\title{
The impact of feeders in closed-loop arrangement on harmonic distortion and power losses
}

\author{
K. Deželak ${ }^{1}$, M. Rošer ${ }^{2}$, R. Škof ${ }^{2}$, T. Kastelic ${ }^{3}$ and G. Štumberger ${ }^{1}$ \\ ${ }^{1}$ University of Maribor \\ Faculty of Electrical Engineering and Computer Science \\ Smetanova 17, 2000 Maribor (Slovenia) \\ Phone/Fax number:+386 2220 7180, e-mail: klemen.dezelak@uni-mb.si, gorazd.stumberger@uni-mb.si, \\ ${ }^{2}$ Elektro Celje d.d. \\ Vrunčeva 2a, 3000 Celje (Slovenia) \\ Phone/Fax number:+386 3420 1000, e-mail: miran.roser@elektro-celje.si, robert.skof@elektro-celje.si \\ ${ }^{3}$ Elektro Primorska d.d. \\ Erjavčeva 22, 5000 Nova Gorica (Slovenia) \\ Phone/Fax number:+386 5339 6700, e-mail: tomaz.kastelic@elektro-primorska.si
}

\begin{abstract}
This paper deals with the power losses and power quality in the medium-voltage distribution network considering feeders in the open- and closed-loop arrangements. The feeders in the open-loop arrangement are changed to the closed-loop arrangement with the aim to improve reliability of the power supply. Apart from that the power quality could be increased, while power losses could be decreased. In the case study the feeders supply loads, while in some cases loads are replaced with the renewable source of energy.
\end{abstract}

\section{Key words}

Medium-voltage distribution network, loop operation, losses, harmonic components.

\section{Introduction}

In the medium-voltage networks, the feeder in closed-loop arrangements are often used to improve voltage profiles in the feeders with distributed generation, to reduce the power losses caused by electric power transmission or to improve reliability and power quality of the power supply [1] - [5]. According to [2], the closed-loop arrangements of the feeders can be classified as the loops of the type I, type II and type III.

Paper [2] deals with the unbalance analysis and with the feasibility study for upgrading the feeders from radial open-loop arrangement to the closed-loop arrangement, while voltage characteristics in a distribution system with distributed generation are presented in [3]. Paper [4] investigates the possibility for reduction of line losses by upgrading the distribution system from radial to closedloop arrangement of feeders.

This work focuses on the loops of type II in mediumvoltage distribution networks [1] and [2]. A special attention is dedicated to the power quality and power losses evaluation in distribution network with the feeders in the open- and closed-loop arrangement. In that way both feeders, that form the closed-loop, are sized in such a way that they can supply all loads on both feeders even when they are supplied only from the one side.

\section{Medium-voltage distribution network}

The discussed medium-voltage network is shown in Fig. 1. It consists of a substation RTP Krško with two 110 $\mathrm{kV} / 20 \mathrm{kV}$ transformers TR I and TR II. They supply two busbars marked by S1 and S2. Measurement points are denoted by M, LD stand for loads, SW is switch, while RS means the renewable source of energy. Voltages and currents are observed also at the high voltage (HV) side of the transformer and at the load LD1, respectively, while RS1 is the renewable source.

With the dynamic model of the discussed system, both, the total harmonic distortion and power losses are analysed, firstly for the open-loop and secondly for the closed-loop arrangement of feeders. The switch that is closing at specific time causes different arrangements of feeders. In that way the harmonic distortion for open- and closed-loop arrangement of feeders and power losses for 
open-loop and closed-loop arrangement of feeders are analyzed.

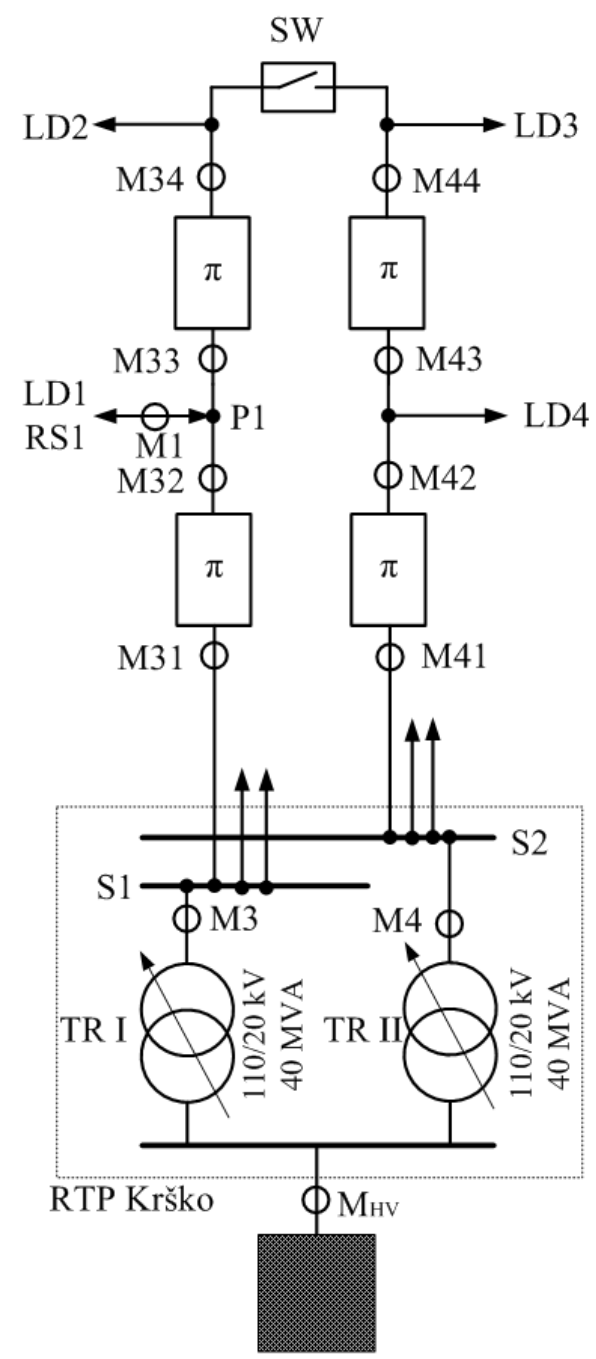

$110 \mathrm{kV}$

Fig. 1. Schematic presentation of discussed system

\section{A. Harmonic distortion calculations}

In this subsection the renewable source of energy RS1 is connected to grid at the point P1, while the load LD1 is equal to zero. In that sense Fig. 2 shows three powers in all three phases $P_{1}, P_{2}$ and $P_{3}$. The renewable source of energy included into the grid causes different higher order harmonic components at the inclusion point $\mathrm{P} 1$, while the feeder at S2 is loaded with linear loads. The switch SW was changed from the open to the closed position at time $t$ $=0.4 \mathrm{~s}$.

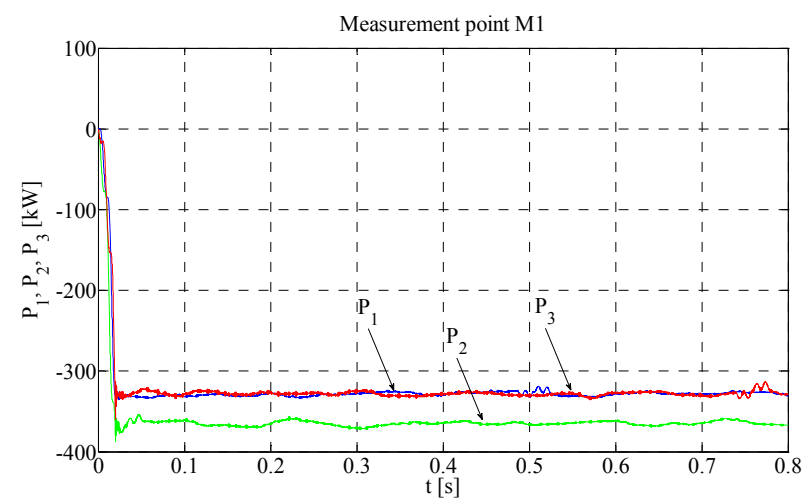

Fig. 2. Power at measurement point M1 (RS1)

The harmonic distortion in currents at the measurement points M31 and M41 are shown in Figs. 3 - 6 for the open-loop and closed-loop arrangements of feeders.

Table 1 shows numerical values of the root mean square (rms) values for voltages and currents in the first phase $\left(U_{\mathrm{rms} 1}, I_{\mathrm{rms} 1}\right)$, power in the first phase $P_{1}$ and total harmonic distortion THD in currents and voltages in the first phase $\left(T H D_{\mathrm{i} 1}, T H D_{\mathrm{u} 1}\right)$. All together the results are shown for five different measurement points (M3, M31, M4, M41 and $\mathrm{M}_{\mathrm{HV}}$ ).

Table I. - Numerical values of $U, I, P$ and $T H D$ for the openand closed-loop arrangement of feeders

\begin{tabular}{|c|c|c|c|}
\hline \multicolumn{2}{|c|}{ Measurement } & Open & Closed \\
\hline \multirow[t]{5}{*}{ M3 } & $U_{\mathrm{rms} 1}[\mathrm{kV}]$ & 12,16365 & 12,18301 \\
\hline & $T H D_{\mathrm{u} 1}[\%]$ & 0,398998 & 0,139603 \\
\hline & $I_{\mathrm{rms} 1}[\mathrm{~A}]$ & 196,2274 & 256,5816 \\
\hline & $T H D_{\mathrm{i} 1}[\%]$ & 0,454397 & 0,140997 \\
\hline & $P_{1}[\mathrm{MW}]$ & 2,369586 & 3,12511 \\
\hline \multirow[t]{5}{*}{ M31 } & $U_{\text {rms1 }}[\mathrm{kV}]$ & 12,16365 & 12,18301 \\
\hline & $T H D_{\mathrm{u} 1}[\%]$ & 0,398998 & 0,139603 \\
\hline & $I_{\mathrm{rms} 1}[\mathrm{~A}]$ & 13,57344 & 75,82212 \\
\hline & $T H D_{\mathrm{i} 1}[\%]$ & 8,430787 & 0,584337 \\
\hline & $P_{1}[\mathrm{MW}]$ & 0,155556 & 0,90288 \\
\hline \multirow[t]{5}{*}{ M4 } & $U_{\text {rms1 }}[\mathrm{kV}]$ & 12,115 & 12,0893 \\
\hline & $T H D_{\mathrm{u} 1}[\%]$ & 0,043732 & 0,140557 \\
\hline & $I_{\mathrm{rms} 1}[\mathrm{~A}]$ & 413,5847 & 354,7476 \\
\hline & $T H D_{\mathrm{i} 1}[\%]$ & 0,011729 & 0,120934 \\
\hline & $P_{1}[\mathrm{MW}]$ & 4,990925 & 4,233001 \\
\hline \multirow[t]{5}{*}{ M41 } & $U_{\mathrm{rms} 1}[\mathrm{kV}]$ & 12,115 & 12,0893 \\
\hline & \begin{tabular}{|l}
$T D_{\mathrm{u} 1}[\%]$ \\
\end{tabular} & 0,043732 & 0,140557 \\
\hline & $I_{\mathrm{rms} 1}[\mathrm{~A}]$ & 57,06966 & 29,6493 \\
\hline & $T H D_{\mathrm{i} 1}[\%]$ & 0,140042 & 2,13721 \\
\hline & $P_{1}[\mathrm{MW}]$ & 0,682958 & $-0,05643$ \\
\hline \multirow[t]{5}{*}{$\mathrm{M}_{\mathrm{HV}}$} & $U_{\mathrm{rms} 1}[\mathrm{kV}]$ & 59,7315 & 59,73349 \\
\hline & $T H D_{\mathrm{u} 1}[\%]$ & 0,04158 & 0,022193 \\
\hline & $I_{\mathrm{rms} 1}[\mathrm{~A}]$ & 170,8765 & 170,7658 \\
\hline & $T^{T H D_{\mathrm{i} 1}[\%]}$ & 0,103096 & 0,093804 \\
\hline & $P_{1}[\mathrm{MW}]$ & 10,03763 & 10,03613 \\
\hline
\end{tabular}



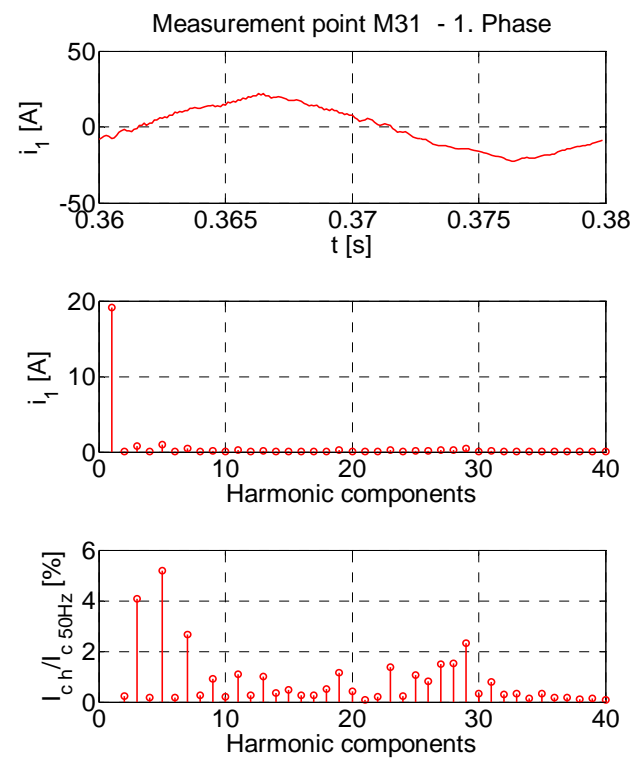

Fig. 3. Harmonic distortion at M31 for the open-loop arrangement of feeders
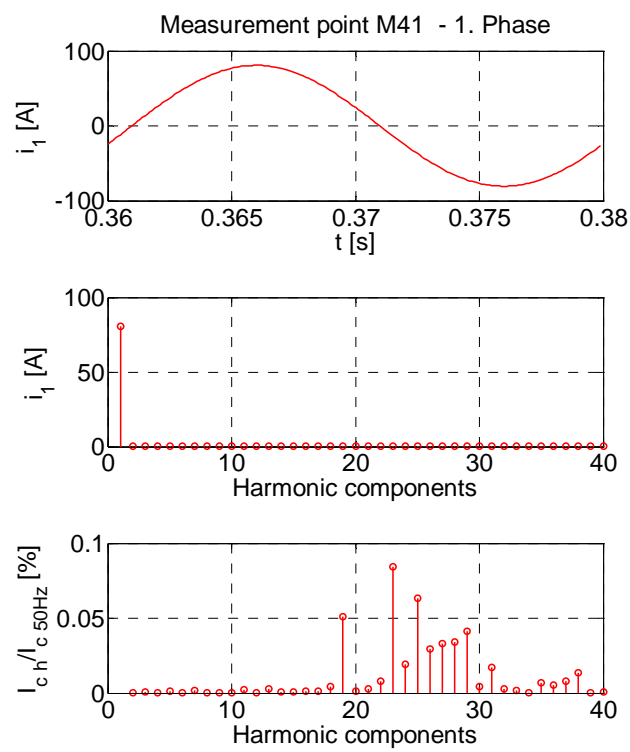

Fig. 4. Harmonic distortion at M41 for the open-loop arrangement of feeders
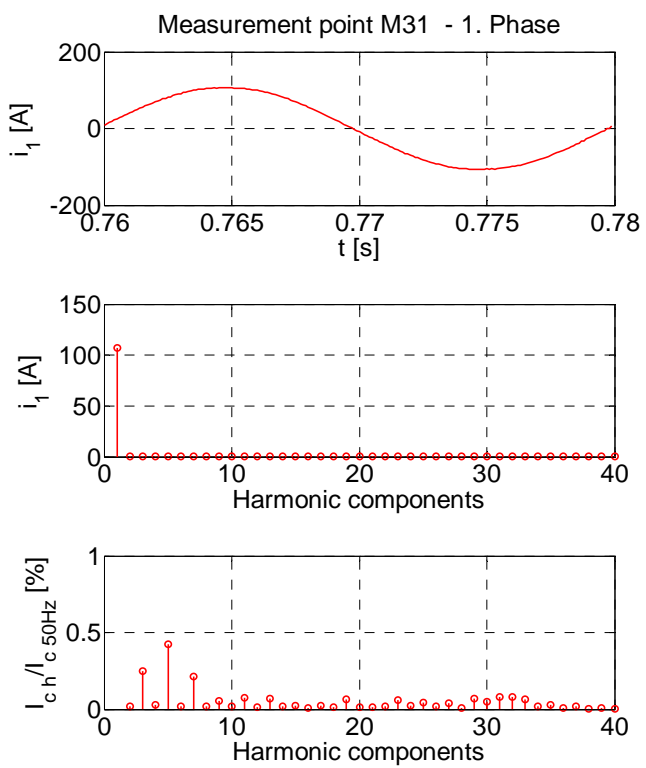

Fig. 5. Harmonic distortion at M31 for the closed-loop arrangement of feeders
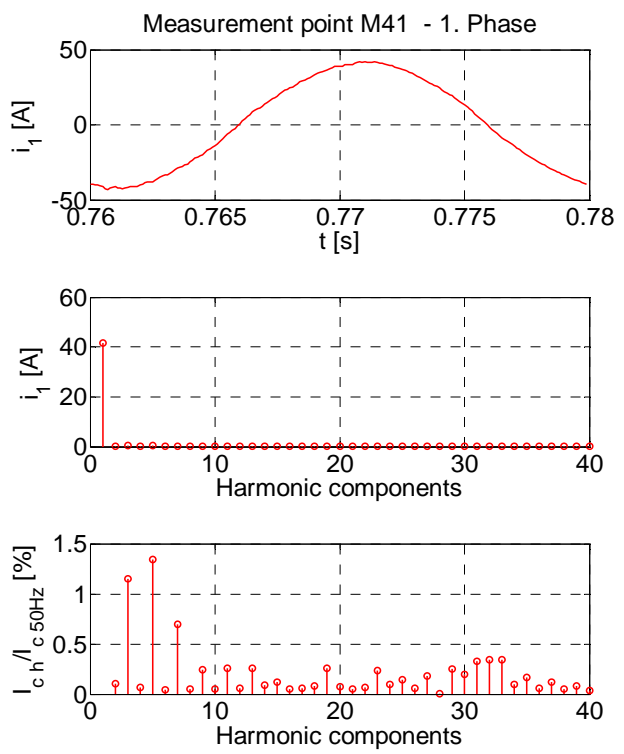

Fig. 6. Harmonic distortion at M41 for the closed-loop arrangement of feeders

The results presented show that both total harmonic distortions at $\mathrm{S} 1$ feeder $\left(T H D_{\mathrm{i} 3}, T H D_{\mathrm{u} 3}\right)$, in the closedloop arrangement, are improved. In that sense the current and voltage THDs decrease from approximately $0.4 \%$ to $0.14 \%$. Even better improvement is reached in the current at the measurement point M31 that is closer to the point P1 (from $8,4 \%$ to $0,58 \%$ ). At the other side $\mathrm{S} 2$ the total harmonic distortions are increased from approximately $0 \%$ to $0.14 \%$ for voltages and to $2.1 \%$ for currents.

The results at the high voltage $\left(\mathrm{M}_{\mathrm{HV}}\right)$ side, before and after the switch closing, show improvement in the total harmonic distortion for the closed-loop arrangements of feeders. 


\section{B. Power losses evaluations}

In this subsection the power losses, on the medium voltage overhead power lines, are observed. Firstly for the openloop and secondly for the closed-loop arrangement of feeders. Different arrangement of feeders is caused by the switch SW closing at $t=0.5 \mathrm{~s}$. Within calculations the load LD1 is connected to the grid, while renewable source of energy RS1 is not connected. In that sense Fig. 7 shows the three powers in all three phases $P_{1}, P_{2}$ and $P_{3}$ at the measurement point M1 (Fig. 1).

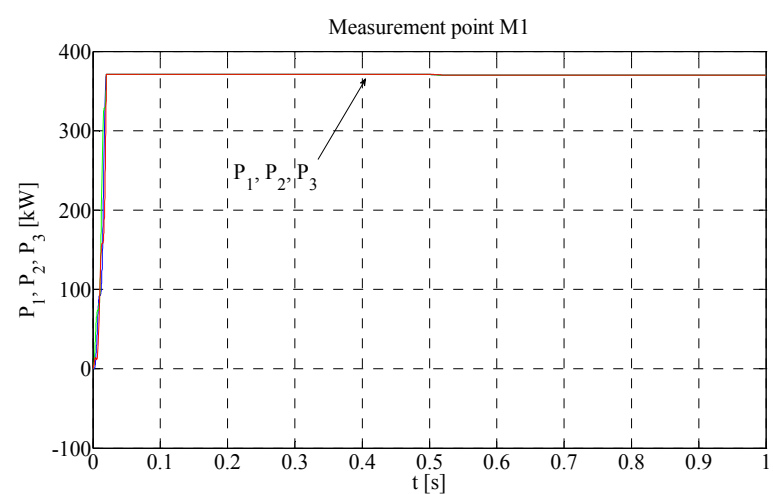

Fig. 7. Power at measurement point M1 (LD1)

Fig. 8 shows the total power losses $\Delta P(1)$ within the openloop (from $t=0 \mathrm{~s}$ to $t=0.5 \mathrm{~s}$ ) and closed-loop (from $t=$ $0.5 \mathrm{~s}$ to $t=1 \mathrm{~s}$ ) arrangement of feeders. In (1) the power losses at $\mathrm{S} 1$ feeder are denote with $\Delta P_{\mathrm{S} 1}$, while $\Delta P_{\mathrm{S} 2}$ stands for the power losses at S2 feeder.

$$
\Delta P=\Delta P_{\mathrm{s} 1}+\Delta P_{\mathrm{s} 2}
$$

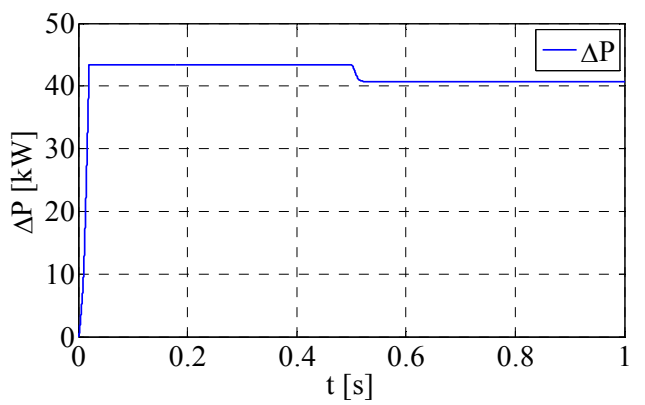

Fig. 8. Total power losses for open-loop and closed-loop arrangement of feeders

It is obvious that the total power losses are reduced (from $43 \mathrm{~kW}$ to $40 \mathrm{~kW}$ ) after the changing operation from opento closed-loop. The reason is that the power losses at S2 feeder $\Delta P_{\mathrm{S} 2}$ are decreased more than the power losses at S1 feeder $\Delta P_{\mathrm{S} 1}$ are increased (Fig. 9).

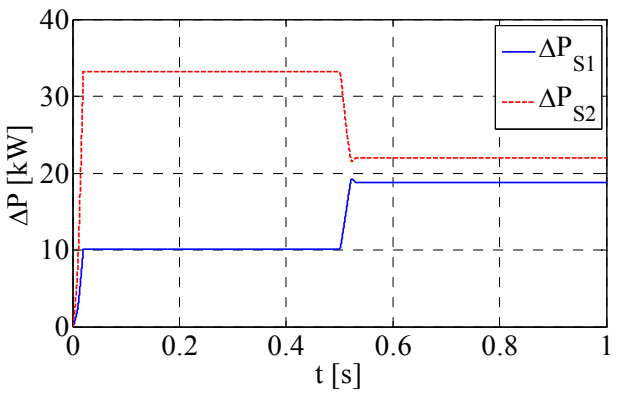

Fig. 9. Power losses for open-loop and closed-loop arrangement of feeders

\section{Conclusion}

The paper deals with the open- and closed-loop arrangement of feeders in the medium-voltage distribution network. The two connected feeders, are supplied from the two different transformers, located in the same substation, therefore they form the loop of type II. In the paper presented results show improvement in power quality and power losses in case of closed-loop arrangement of feeders. Additionally, the closed-loop arrangement can substantially improve the voltage profiles and the power supply reliability, however, before closed-loop arrangement of feeder can be implemented, problems related with proper realisation of protection should be solved.

\section{References}

[1] G. Štumberger, K. Deželak, M. Rošer, R. Škof, T. Kastelic, Medium-voltage distribution feeders in open-loop and closedloop arrangement, Proceedings of the International conference on renewable energies and power quality (ICREPQ' 12), 2012, Spain.

[2] T. H. Chen, W. T. Huang, J. C. Gu, G. C. Pu, Y. H. Hsu, T. Y. Guo, Feasibility Study of Upgrading Primary Feeders from Radial and Open-Loop to Narmally Closed-Loop Arrangement, IEEE Transactions on Power Systems, vol. 19, no. 3, pp. 1308 1316, 2004.

[3] N. Okada, H. Kobayashi, K. Takigawa, M. Ichikawa, K. Kurokawa, Loop power flow control and voltage characteristics of distribution system for distributed generation including pv system, 3rd World Conference on Photovoltoic Energy Conversion, May 11-18, Osaka. Japan, pp. 2284-2287, 2003.

[4] W. T. Huang, S. T. Chen, Line Loss Reduction by Distribution Sytem Upgrading from Radial to Normally ClosedLoop Arrangement, Ninth International Conference on Hybrid Intelligent Systems, Washington, USA, vol. 3, pp. 334 - 339, 2009.

[5] W. T. Huang, T. H. Chen, G. C. Pu, Y. F. Hsu, T. Y. Guo, Assessment of Upgrading Existing Primary Feeders from Radial to Normally Closed Loop Arrangement, IEEE Transmission and Distribution Conference and Exhibition, 3, 2002. 